\section{The individual and neighborhood factors associated with the use of emergency medical services in patients with ST-elevation myocardial infarction}

\author{
Hanzo Choi, Won Chul Cha, Ik Joon Jo, Jin-Ho Choi, Min Seob Sim, \\ Taegun Shin
}

Department of Emergency Medicine, Samsung Medical Center, Sungkyunkwan University School of Medicine, Seoul, Korea

Objective The utilization of emergency medical services (EMS) varies widely among communities. In this study, we aimed to evaluate the relationship between the use of EMS by patients with ST-elevation myocardial infarction (STEMI) and the individual and neighborhood characteristics of these patients.

Methods We performed a secondary analysis of data from the Cardiovascular Disease Surveillance project, which included patients diagnosed with STEMI at 29 emergency centers in South Korea. Our analysis included only patients living in Seoul, and the primary outcome measured was the use of EMS. While the clinical variables of the patients were collected from the Cardiovascular Disease Surveillance registry, the 2010 National Census data was used to identify neighborhood variables such as population density, income, age, and residence type. We used a 3-level hierarchical logistic regression to estimate the effects of neighborhood-level factors on EMS use by individual patients.

Results We evaluated 1,634 patients with STEMI from 2007 to 2012. The neighborhoods were grouped into 25 counties. The regional rates of EMS use varied from 18.3\% to 46.5\%. The final adjusted logistic model revealed that the use of EMS was significantly associated with the average number of households (neighborhood level factor) and symptoms of syncope, cardiac arrest, and history of cardiovascular disease (individual level factors).

Conclusion The individual levels factors had a greater influence on the use of EMS compared to the neighborhood-level factors.

Keywords Emergency medical services; Residence characteristics; Acute coronary syndrome; Censuses; Logistic models

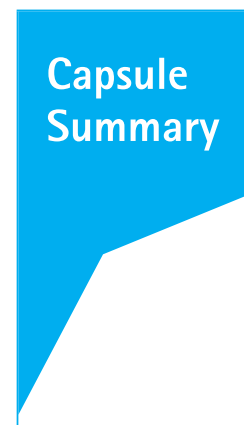

What is already known

Neighborhood level factors were significantly associated with individual emergency medical services (EMS) use in stroke and other disease other than ST-elevation myocardial infarction.

What is new in the current study

Neighborhood level factors such as average number of households were significantly associated with individual EMS use. Although neighborhood-level factors were associated with EMS use, individual level factors had more influences on EMS use.
eISSN: 2383-4625

Received: 22 September 2019

Revised: 6 January 2020

Accepted: 6 January 2020

Correspondence to: Won Chul Cha Department of Emergency Medicine, Samsung Medical Center,

Sungkyunkwan University School of Medicine, 81 Irwon-ro, Gangnam-gu, Seoul 06351, Korea

E-mail:wc.cha@samsung.com ORCID

https://orcid.org/0000-0002-2778-2992

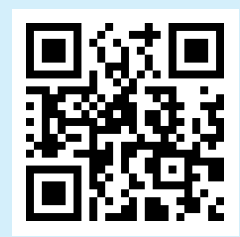

How to cite this article:

Choi H, Cha WC, Jo IJ, Choi JH, Sim MS, Shin T. The individual and neighborhood factors associated with the use of emergency medical services in patients with ST-elevation myocardial infarction. Clin Exp Emerg Med 2020;7(4):302-309. https://doi. org/10.15441/ceem.19.083

This is an Open Access article distributed under the terms of the Creative Commons Attribution Non-Commercial License (https:// creativecommons.org/licenses/by-nc/4.0/). 


\section{INTRODUCTION}

The current guidelines state the importance of establishing regionalized cardiac care systems to reduce the mortality and morbidity associated with acute myocardial infarction. ${ }^{1,2}$ It is well known that restoring coronary perfusion within a short delay period is essential for better outcomes in such cases. ${ }^{3-5}$ In order to achieve this goal, a well-organized setup, including efficient emergency medical services (EMS) and high-quality hospital care systems, is crucial. ${ }^{6}$

The first step in establishing organized cardiac care is the recognition of cardiac symptoms by both the patients themselves and bystanders, followed by activation of the EMS system. Timely activation of the EMS system is related to shorter periods of delay and better clinical outcomes in patients with ST-elevation myocardial infarction (STEMI)., ${ }^{78}$ However, the actual rate of EMS activation is very low in South Korea, and community-level interventions to increase this have yielded different outcomes. ${ }^{9,10}$

In order to carry out effective and efficient community-level interventions, it is, therefore, necessary to identify areas with increased risk within the community. Recent studies have revealed that neighborhood characteristics are significantly related to the rate of EMS activation in multiple acute diseases such as cardiac arrest and stroke. ${ }^{11-13}$ This is consistent with previous studies that found that neighborhood factors such as the economic state and proportion of elderly individuals are associated with the rate of EMS activation. ${ }^{14,15}$

In this study, we aimed to identify the individual and neighborhood characteristics of patients with STEMI and evaluate the association of these factors with EMS activation rates.

\section{METHODS}

\section{Study design}

This cross-sectional observational study was based on the Cardiovascular Disease Surveillance (CAVAS) project and on the 2010 Korea National Census database. The CAVAS project, sponsored by the Korean Centers for Disease Control and the National Emergency Management Agency since 2006, is a nationwide, observational database of all EMS associated with out-of-hospital cardiac arrests and acute cardiovascular and cerebrovascular disease, diagnosed in the emergency department (ED) within 1 week of the onset of symptoms. ${ }^{16-18}$

The data on patients admitted or discharged with acute cardiovascular and cerebrovascular disease (International Classification of Diseases 10 codes: I21.0-I21.9, I60.0-I60.9, I61.0-I61.9, and I63.0-I63.9, I64) from 29 participating EDs in 12 cities and provinces across South Korea were prospectively collected through in-depth hospital medical record reviews. The collected data included demographic (age, sex, and address), socioeconomic (insurance status, education, and occupation), and risk factors (hypertension, diabetes, dyslipidemia, chronic renal failure, cardiovascular, and cerebrovascular disease, smoking, and alcohol use) data. Trained reviewers abstracted the data from the medical records.

The study protocol was approved by the institutional review board of Samsung Medical Center (2016-04-060).

\section{Setting}

Seoul, the capital of South Korea, is a metropolis with 25 counties, each called "gu". In 2010, the total population of Seoul was approximately 9.5 million, and the average population density was 17,142 inhabitants $/ \mathrm{km}^{2}$. South Korea has a single-tiered and government-based EMS system. In 2010, the Korean EMS was operated by the fire department from 16 provincial headquarters with 1,349 ambulances and served a population of about 50 million. ${ }^{16}$ The primary EMS agency responds to all 119 calls for medical emergencies. The EMS use is free of cost, and the average response time is 6.5 minutes. $^{19}$

\section{Selection of participants}

The CAVAS project included patients who were diagnosed with STEMI and non-STEMI at 29 emergency centers in South Korea between January 2007 and December 2012. We targeted participants who lived in Seoul, were adults older than 18 years of age, and visited the above mentioned 29 emergency centers. A STEMI diagnosis was established for patients who showed ST-segment elevation following an electrocardiogram (ECG) in the ED, but not for patients who showed the same during ECG performed at the prehospital phase. This was because the ECG results could not be analyzed directly at the prehospital phase.

Methods of measurement, data collection, and processing Trained abstractors collected individual-level information from source documents at the hospitals using standard procedures. The project manager and study physicians checked the data quality.

For neighborhood characteristics, we used the summary statistics from the 2010 Korea National Census data for the residence of each patient. We obtained the address of residence from the medical records of the patients.

\section{Outcomes and measurements}

The primary outcome evaluated was the arrival of patients at the final hospital following the activation of EMS. Neighborhood-level 
variables included population density, the proportion of females, the proportion of individuals older than 65 years, the proportion of apartments, the average number of households, and the average income for each county. Further details on the methodology used in the census can be found at the website of the Statistics Korea. ${ }^{20}$ Previous studies have shown that high population densities and high proportions of females and elderly people lead to higher EMS utilizations..$^{15,21,22}$ Residences in high-rise buildings have longer EMS response times. ${ }^{23}$ Therefore, the proportion of apartments in the area was included in the analysis to assess its association with EMS utilizations.

Individual-level variables included age, sex, level of education, type of health insurance, main symptoms, smoking history, and past medical history.

\section{Primary data analysis}

We analyzed the descriptive statistics of the study population using means with standard deviations, medians with interquartile ranges, ranges, and proportions. We have graphically depicted the proportion of EMS use for each county, using ArcGIS ver. 10.1 (Environmental Systems Research Institute, Redlands, CA, USA).

All neighborhood-level variables were set as continuous variables. The population density was categorized into levels, with every succeeding level showing an increase of 1,000 people. Similarly, average income was categorized into levels, with every succeeding level showing an increase of 1 million Korean won. All individual-level variables except age and education level were categorized. Categorical variables included sex (male vs. male), insurance type (national health insurance vs. medical aid), main symptoms (chest pain, dyspnea, syncope, cardiac arrest, and others), smoking status (current smoker, ex-smoker, and non-smoker), and the past history of diseases (yes vs. no).

To compare between the EMS users and EMS non-users, we used the Student t-test and chi-square test for parametric variables and the Mann-Whitney test for non-parametric variables. To determine the associations of individual-level and neighborhood-level characteristics with EMS use, we used a 3-level L model. This allowed us to account for the nesting of 1,634 patients (level 1) within 25 neighborhoods defined as census tracts (level 2) and 25 random intercepts (level 3). First, we developed a binary logistic regression model using "arrival by EMS (yes vs. no)" as the dependent variable. Next, individual-level (model 1) and neighborhood-level (model 2) characteristics were added as fixed effects, and census tracts were added as random effects to this model in order to examine their independent contributions. The final model was chosen based on the greatest proportion of variance that was explained by individual and neighborhood variables. We also calculated the intra-class correlation coefficients to estimate how much of the variability in the relationship between the outcomes and predictors was explained by correlation within neighborhood variables and how much of the variability was caused by other reasons (differences across patients and random variation).

All statistical analyses were performed using $R$ ver. 3.3.1 (R Foundation for Statistical Computing, Vienna, Austria). P-values were based on a two-sided significance level of 0.05 .

\section{RESULTS}

\section{Characteristics of the study subjects}

From January 2007 to December 2012, the CAVAS project identified 9,989 patients with STEMI. Patients who had no record of their addresses $(n=3,455)$, did not live in Seoul $(n=4,726)$, transferred to a hospital outside Seoul $(n=126)$, and had no record of EMS use $(n=48)$ were excluded from the study. Finally, 1,634 patients were included in this study (Fig. 1).

The average age of the subjects was 62.1 years, and $21.7 \%$ of them were females. The re-transfer rate until the visit to the final hospital was $31.5 \%$.

There are 25 counties in Seoul. The median population density and proportion of females in these counties were 17,142 inhabitants $/ \mathrm{km}^{2}$ and $51.6 \%$, respectively. The median proportion of people aged over 65 years, the average number of households, and the average income were 10.3\%, 2.6, and 46.44 million won, respectively (Table 1).

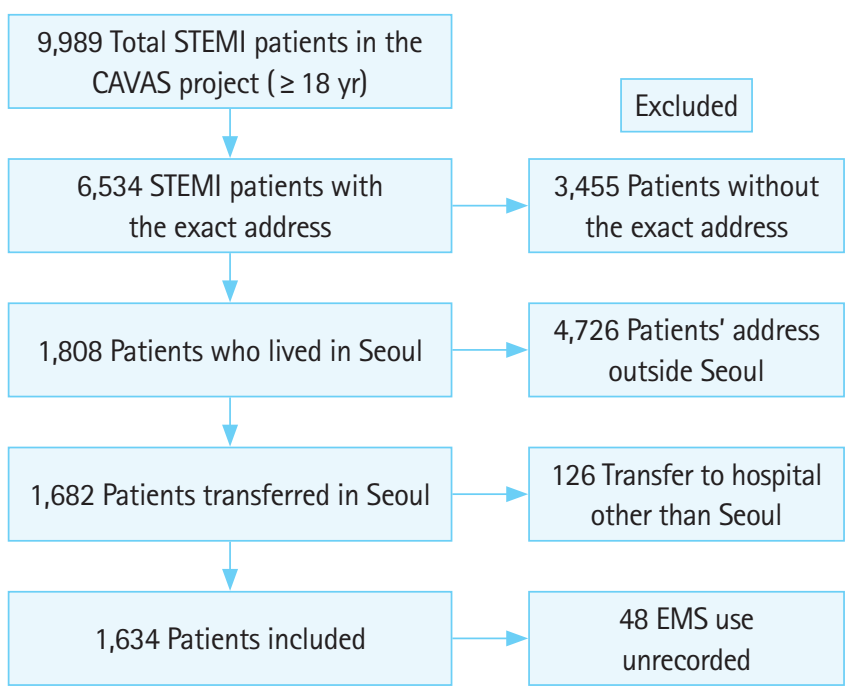

Fig. 1. Flow chart of enrolled ST- patients. STEMI, ST-elevation myocardial infarction; CAVAS, Cardiovascular Disease Surveillance; EMS, emergency medical services. 
Table 1. Characteristics of ST-elevation myocardial infarction patients in Seoul, 2007 to 2012

\begin{tabular}{lcc}
\hline Variable & \multicolumn{1}{c}{ Value } & $\begin{array}{c}\text { No. of } \\
\text { missing }\end{array}$ \\
\hline Individual ( $\mathrm{n}=1,634)$ & & \\
Age (yr) & $62.1 \pm 13.2$ & 0 \\
Sex, female & $355(21.7)$ & 0 \\
Level of education & $12(9-16)$ & 52 \\
Medical insurance (vs. Medicaid) & $1,576(96.5)$ & 0 \\
Smoking status & & 2 \\
$\quad$ Current-smoker & $695(42.5)$ & \\
Ex-smoker & $292(17.9)$ & \\
$\quad$ Non-smoker & $645(39.5)$ & 9 \\
Past history of diseases & & 9 \\
Diabetes mellitus & $443(27.1)$ & 17 \\
Hypertension & $822(50.3)$ & 13 \\
Hyperlipidemia & $173(10.6)$ & 15 \\
Kidney disease & $80(4.9)$ & 13 \\
Coronary artery disease & $273(16.7)$ & 0 \\
Stroke/TIA & $110(6.7)$ & 0 \\
Transferred from another hospital & $515(31.5)$ & 0 \\
Neighborhood ( $\mathrm{n}=23)$ & & 0 \\
Population density & $17,142(13,434-19,389)$ & 0 \\
Percentage of women & $51.6(50.9-51.9)$ & 0 \\
Percentage of people aged $>65$ yr & $10.3(8.7-11.4)$ & 0 \\
Percentage of apartments & $53.8(46.8-65.4)$ & 0 \\
Average number of households & $2.6(2.6-2.8)$ & \\
Average income (10,000 Korean won) & $4,644(4,340-4,949)$ & 0 \\
\hline & & \\
\hline
\end{tabular}

Values are presented as mean \pm standard deviation, number (\%), or median (interquartile range).

TIA, transient ischemic attack.

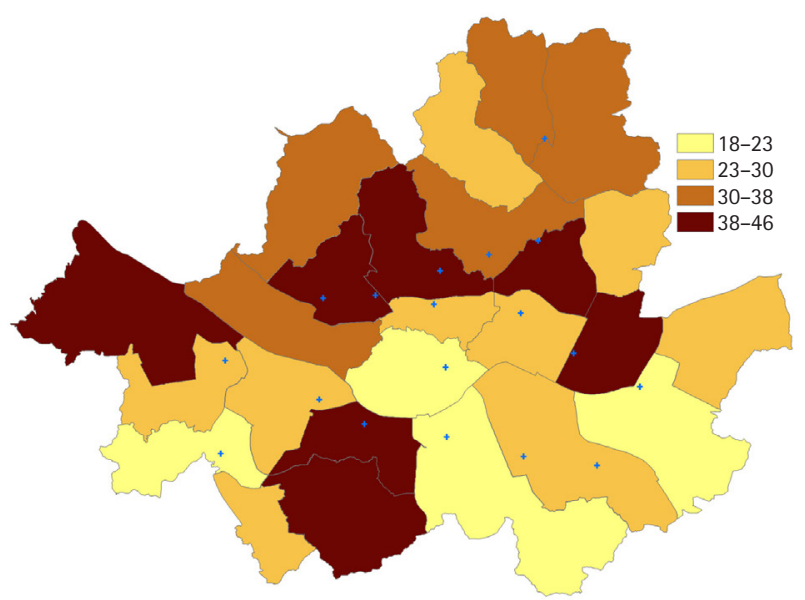

Fig. 2. Percentage of emergency medical services use in Seoul. Seoul has 25 counties, each called "gu". Blue points indicate senior general hospitals.

\section{Main results}

The average rate of EMS use in Seoul was 33.9\% among the included patients, while the regional rates varied from $18.3 \%$ to
Table 2. Comparison of individual factors between the EMS group and non-EMS group $(n=1,634)$

\begin{tabular}{|c|c|c|c|}
\hline Variable & $\begin{array}{c}\text { EMS } \\
(n=577)\end{array}$ & $\begin{array}{l}\text { Non-EMS } \\
(n=1,057)\end{array}$ & P-value \\
\hline Age (yr) & $62.6 \pm 12.9$ & $61.7 \pm 13.4$ & $0.192^{\mathrm{a})}$ \\
\hline Sex, female & $131(22.7)$ & $224(21.2)$ & $0.479^{b)}$ \\
\hline $\begin{array}{l}\text { Medical aid (vs. national health } \\
\text { insurance) }\end{array}$ & $30(5.2)$ & $28(2.6)$ & $0.008^{b)}$ \\
\hline Symptoms & & & $<0.001^{\text {b) }}$ \\
\hline Chest pain & $462(80.1)$ & 925 (87.5) & \\
\hline Dyspnea & $25(4.3)$ & $51(4.8)$ & \\
\hline Syncope & $19(3.3)$ & $11(1.0)$ & \\
\hline Cardiac arrest & $43(7.5)$ & $11(1.0)$ & \\
\hline Others & $28(4.9)$ & $59(5.6)$ & \\
\hline Smoking status & & & $0.575^{b)}$ \\
\hline Current-smoker & $258(44.7)$ & $437(41.3)$ & \\
\hline Ex-smoker & $99(17.2)$ & 195 (18.5) & \\
\hline Non-smoker & $220(38.1)$ & $425(40.2)$ & \\
\hline \multicolumn{4}{|l|}{ Past history of diseases } \\
\hline Diabetes mellitus & $152(26.3)$ & $291(27.5)$ & $0.606^{\text {b) }}$ \\
\hline Hypertension & $275(47.7)$ & $547(51.8)$ & $0.114^{b)}$ \\
\hline Hyperlipidemia & $67(11.6)$ & $106(10.0)$ & $0.320^{\text {b) }}$ \\
\hline Kidney disease & $31(5.4)$ & $49(4.6)$ & $0.509^{b)}$ \\
\hline Coronary artery disease & $139(24.1)$ & $134(12.7)$ & $<0.001^{\text {b) }}$ \\
\hline Stroke/TIA & $48(8.3)$ & $62(5.9)$ & $0.059^{b)}$ \\
\hline Transferred from another hospital & $48(8.3)$ & $463(43.8)$ & $<0.001^{\text {b) }}$ \\
\hline Level of education (yr) & $12(6-16)$ & $12(9-16)$ & $0.036^{\mathrm{c})}$ \\
\hline $\begin{array}{l}\text { Average time from symptom onset to } \\
\text { arrival at hospital (min) }\end{array}$ & $48(25-120)$ & $206(71-691)$ & $<0.001^{\text {c) }}$ \\
\hline
\end{tabular}

Values are presented as mean \pm standard deviation, number (\%), or median (interquartile range).

EMS, emergency medical services; TIA, transient ischemic attack.

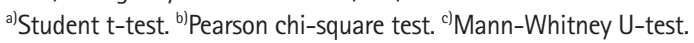

46.5\% (Fig. 2). We compared the individual-level variables between the groups of EMS-users and EMS-non-users. The proportion of individuals with medical aid (5.2\% vs. $2.6 \%)$, and main symptoms such as syncope (3.3\% vs. $1 \%)$, cardiac arrest $(7.5 \%$ vs. $1 \%)$ and a history of cardiovascular disease (24.1\% vs. $12.7 \%)$ was higher among the EMS-users when compared to the EMS non-users. However, the EMS-users when compared to the EMS non-users showed the shorter average time from the onset of symptoms to arrival at hospital (48 vs. 206 minutes), and lower retransfer rate until the final hospital visit (8.3\% vs. 43.8\%) (Table 2).

To create the final model, an univariate logistic regression was performed on all the variables that influenced the use of EMS. Significant correlations were observed between the use of EMS and individual variables such as the type of health insurance, level of educations, main symptoms, and history of cardiovascular disease. Among the neighborhood variables, the proportion of females and apartments, the average number of households, and the average income showed significant correlations with the use 


\section{of EMS (Table 3).}

For all the three hierarchical logistic regression models, the intra-class correlation coefficient for the random intercept neighborhood variable was 0.065 , indicating that only $6.5 \%$ of the variation in EMS use was explained by the census tract level while the remaining variation was caused by individual-level factors or

\section{random variations.}

Table 4 shows the final multivariable model and the adjusted odds ratios and 95\% confidence intervals for EMS use. At an individual level, patients who had national health insurance were less likely to use EMS when compared to those who had medical aid. Patients who had symptoms such as syncope and cardiac ar-

Table 3. Univariate logistic model of individual and neighborhood factors associated with emergency medical services use

\begin{tabular}{|c|c|c|c|c|}
\hline Variable & $\mathrm{OR}$ & $95 \% \mathrm{CIL}$ & $95 \% \mathrm{CIU}$ & P-value \\
\hline \multicolumn{5}{|l|}{ Individual level } \\
\hline Age (per 1 year increase) & 1.005 & 0.997 & 1.013 & 0.192 \\
\hline National health insurance vs. medical aid & 0.496 & 0.293 & 0.839 & 0.009 \\
\hline Level of education (per 1 year increase) & 0.976 & 0.953 & 0.999 & 0.045 \\
\hline Dyspnea vs. chest pain & 0.981 & 0.600 & 1.604 & 0.940 \\
\hline Cardiac arrest vs. chest pain & 7.827 & 3.999 & 15.319 & $<0.001$ \\
\hline Others vs. chest pain & 0.950 & 0.598 & 1.510 & 0.829 \\
\hline Current smoker vs. non-smoker & 1.141 & 0.912 & 1.427 & 0.250 \\
\hline Ex-smoker vs. non-smoker & 0.976 & 0.728 & 1.307 & 0.870 \\
\hline History of diabetes mellitus vs. no history of diabetes mellitus & 0.941 & 0.749 & 1.184 & 0.606 \\
\hline History of stroke/TIA vs. no history of stroke/TIA & 1.456 & 0.985 & 2.154 & 0.060 \\
\hline \multicolumn{5}{|l|}{ Neighborhood level } \\
\hline Population density (per 1,000 increase) & 0.997 & 0.974 & 1.022 & 0.832 \\
\hline Percentage of women (per 1 percent increase) & 0.871 & 0.763 & 0.994 & 0.040 \\
\hline Percentage of people aged $>65$ years (per 1\% increase) & 1.059 & 0.992 & 1.131 & 0.085 \\
\hline Percentage of apartments (per 1\% increase) & 0.989 & 0.982 & 0.996 & 0.002 \\
\hline Average number of households & 0.397 & 0.219 & 0.722 & 0.002 \\
\hline Average income (per 1 million Korean won increase) & 0.984 & 0.974 & 0.994 & 0.002 \\
\hline
\end{tabular}

$\mathrm{OR}$, odds ratio; $\mathrm{CIL}$, lower bound of 95\% confidential interval; $\mathrm{CIU}$, upper bound of 95\% confidential interval; $C A D$, coronary artery disease; TIA, transient ischemic attack.

Table 4. Final adjusted logistic model of individual and neighborhood factors associated with emergency medical services use

\begin{tabular}{|c|c|c|c|c|}
\hline Variable & $\mathrm{OR}$ & $95 \% \mathrm{CIL}$ & $95 \% \mathrm{CIU}$ & P-value \\
\hline \multicolumn{5}{|l|}{ Individual level } \\
\hline National health insurance vs. medical aid & 0.536 & 0.307 & 0.935 & 0.028 \\
\hline Level of education (per 1 year increase) & 0.987 & 0.962 & 1.012 & 0.307 \\
\hline Dyspnea vs. chest pain & 0.934 & 0.558 & 1.562 & 0.794 \\
\hline Syncope vs. chest pain & 3.578 & 1.597 & 8.016 & 0.002 \\
\hline Cardiac arrest vs. chest pain & 7.958 & 4.001 & 15.832 & $<0.001$ \\
\hline Others vs. chest pain & 1.016 & 0.627 & 1.647 & 0.948 \\
\hline History of CAD vs. no history of CAD & 2.348 & 1.781 & 3.095 & $<0.001$ \\
\hline \multicolumn{5}{|l|}{ Neighborhood level } \\
\hline Percentage of women (per $1 \%$ increase) & 1.090 & 0.902 & 1.317 & 0.372 \\
\hline Percentage of apartments (per 1\% increase) & 0.995 & 0.984 & 1.005 & 0.330 \\
\hline Average number of households & 0.496 & 0.248 & 0.992 & 0.047 \\
\hline Average income (per 1 million Korean won increase) & 0.987 & 0.969 & 1.004 & 0.138 \\
\hline
\end{tabular}

$\mathrm{OR}$, odds ratio; $\mathrm{CIL}$, lower bound of 95\% confidential interval; $\mathrm{CIU}$, upper bound of 95\% confidential interval; $C A D$, coronary artery disease. 
rest were more likely to use EMS than those who had other symptoms. Patients with coronary artery disease were also more likely to use EMS when compared to those without coronary artery disease. At the neighborhood level, only the average number of households showed a significant association with EMS use; the higher the average number of households, the lower was the EMS utilization rate.

\section{DISCUSSION}

To the best of our knowledge, this is the first study to show an association between neighborhood characteristics and EMS use in patients with STEMI. Among the patients included in our study, only $35 \%$ used EMS, and $43.8 \%$ of those who did not use EMS were retransferred to the final hospital. The time taken to arrive at the final hospital after the onset of symptoms was noticeably shorter in patients who used EMS than in those who did not use EMS (48 vs. 206 minutes, $P<0.001$ ), suggesting that the use of EMS is essential for the final treatment such as fibrinolysis or percutaneous coronary intervention in patients with STEMI. So, what are the factors that influence the use of EMS?

Our final model showed that symptoms such as syncope, cardiac arrest, and history of coronary artery disease (individual-level factors), as well as the average number of households (neighborhood-level factor), were significantly associated with the use of EMS. Previous studies have revealed that the severity of stroke is associated with the likelihood of EMS use. ${ }^{11}$ Although the National Institutes of Health Stroke Scale reflects the severity of symptoms in cases of stroke, there is no accurate measure of the severity of symptoms in cases of STEMI. In this study, we, therefore, decided to use the four most common symptoms of STEMI (chest pain, dyspnea, syncope, and cardiac arrest) to determine the severity. Generally, syncope and cardiac arrest are considered to be more severe symptoms. Consistent with previous studies, we found that the more severe the symptoms of STEMI were, the higher was the rate of EMS use. ${ }^{24}$

Some studies have evaluated the association between EMS use and past medical history. ${ }^{11,24}$ This study showed that patients with STEMI who had a history of coronary artery disease were much more likely to use EMS (odds ratio, 2.47) compared to those without a history of coronary artery disease. However, Meurer et al. ${ }^{11}$ have shown that a past history of stroke did not affect the use of EMS. This could be due to the differences in the outcomes of stroke and STEMI. ${ }^{11}$

At the neighborhood level, only the average number of households affected the use of EMS. As the average number of households in the patient's area increased by 1 , the EMS utilization tended to decrease by $51 \%$ (odds ratio, 0.49). With the increase in the number of households, it was more likely that a patient living near a hospital would be transported directly to the hospital without activating the EMS. In fact, the interpretation of results at the neighborhood level may not be easy. Among the studies that have evaluated the association between neighborhood characteristics and EMS use, not many have assessed the association between the average number of households in the region and the use of EMS. Earnest et al. ${ }^{25}$ postulated that the inverse relationship between the number of households and trauma-related EMS calls could be related to the presence of caregivers and family members for the management of vulnerable elders and young children. However, more studies will be needed to confirm our findings.

Some studies have emphasized the potential influence of neighborhood factors on EMS use. For example, Sasson et al. ${ }^{13}$ showed that poor neighborhoods exhibited lower rates of bystander cardiopulmonary resuscitation. Treno et al. ${ }^{26}$ showed that neighborhood-focused alcohol abuse intervention could result in reduced crime, increased EMS use, and less number of injuries. Based on the significantly higher odds ratio for individual level factors compared to the neighborhood factors, our results suggest that in patients with STEMI, the former have a stronger influence on the rate of EMS use compared to the later.

Even with some limitations, this nationwide study demonstrates an association between neighborhood characteristics and the use of EMS, which is a valuable point to consider while initiating community programs to facilitate early interventions for patients with STEMI.

This study has important limitations. First, only one city was included in the study. Seoul is the biggest city in South Korea and has characteristics that are very different from other cities. These include the total population, population density, and percentage of apartments. Second, we included only urban areas in our study. Thus, our results may not be completely applicable to rural areas. However, during the survey, there were rural areas where patients with STEMI never used EMS. Since the EMS system is very different in regions other than Seoul, it is difficult to study it in other areas, including the rural areas. As a result, our conclusions may not be applicable to the entire nation, especially to the rural areas. Third, we used the census data for only 2010. It would be nice to evaluate the data for every year, but we could not do so because of the updates every 5 years. We used census data for 2010, which was the midpoint between 2007 and 2012.

In conclusion, interventions to improve EMS use for patients with STEMI should focus on individuals and not on the neighborhoods. From a neighborhood perspective, it would be beneficial 
to educate people and develop local policies to increase the use of EMS in areas with a large number of households.

\section{CONFLICT OF INTEREST}

No potential conflict of interest relevant to this article was reported.

\section{REFERENCES}

1. American Heart Association. 2010 American Heart Association guidelines for cardiopulmonary resuscitation and emergency cardiovascular care. Circulation 2010;122:s639-946.

2. Le May MR, Davies RF, Dionne R, et al. Comparison of early mortality of paramedic-diagnosed ST-segment elevation myocardial infarction with immediate transport to a designated primary percutaneous coronary intervention center to that of similar patients transported to the nearest hospital. Am J Cardiol 2006;98:1329-33.

3. Armstrong PW, Westerhout CM, Welsh RC. Duration of symptoms is the key modulator of the choice of reperfusion for STelevation myocardial infarction. Circulation 2009;119:1293303.

4. Ho AF, Pek PP, Fook-Chong S, et al. Prehospital system delay in patients with ST-segment elevation myocardial infarction in Singapore. World J Emerg Med 2015;6:277-82.

5. Lee SY, Ro YS, Shin SD, et al. Interaction effects between highly-educated neighborhoods and dispatcher-provided instructions on provision of bystander cardiopulmonary resuscitation. Resuscitation 2016;99:84-91.

6. Sorensen JT, Maeng M. Regional systems-of-care for primary percutaneous coronary intervention in ST-elevation myocardial infarction. Coron Artery Dis 2015;26:713-22.

7. Choi SW, Shin SD, Ro YS, Song KJ, Lee YJ, Lee EJ. Effect of emergency medical service use and inter-hospital transfer on time to percutaneous coronary intervention in patients with ST elevation myocardial infarction: a multicenter observational study. Prehosp Emerg Care 2016;20:66-75.

8. Mathews R, Peterson ED, Li S, et al. Use of emergency medical service transport among patients with ST-segment-elevation myocardial infarction: findings from the National Cardiovascular Data Registry Acute Coronary Treatment Intervention Outcomes Network Registry-Get With The Guidelines. Circulation 2011;124:154-63.

9. Blohm M, Hartford M, Karlson BW, Karlsson T, Herlitz J. A media campaign aiming at reducing delay times and increasing the use of ambulance in AMI. Am J Emerg Med 1994;12:315-8.
10. Luepker RV, Raczynski JM, Osganian S, et al. Effect of a community intervention on patient delay and emergency medical service use in acute coronary heart disease: the Rapid Early Action for Coronary Treatment (REACT) Trial. JAMA 2000;284: 60-7.

11. Meurer WJ, Levine DA, Kerber KA, et al. Neighborhood influences on emergency medical services use for acute stroke: a population-based cross-sectional study. Ann Emerg Med 2016; 67:341-8.

12. Sasson C, Keirns CC, Smith D, et al. Small area variations in out-of-hospital cardiac arrest: does the neighborhood matter? Ann Intern Med 2010;153:19-22.

13. Sasson $C$, Magid DJ, Chan $P$, et al. Association of neighborhood characteristics with bystander-initiated CPR. N Engl J Med 2012;367:1607-15.

14. Govindarajan A, Schull M. Effect of socioeconomic status on out-of-hospital transport delays of patients with chest pain. Ann Emerg Med 2003;41:481-90.

15. Platts-Mills TF, Leacock B, Cabanas JG, Shofer FS, McLean SA. Emergency medical services use by the elderly: analysis of a statewide database. Prehosp Emerg Care 2010;14:329-33.

16. Ahn KO, Shin SD, Suh GJ, et al. Epidemiology and outcomes from non-traumatic out-of-hospital cardiac arrest in Korea: a nationwide observational study. Resuscitation 2010;81:97481.

17. Shin SD, Ahn KO, Song KJ, Park CB, Lee EJ. Out-of-hospital airway management and cardiac arrest outcomes: a propensity score matched analysis. Resuscitation 2012;83:313-9.

18. Shin SD, Suh GJ, Ahn KO, Song KJ. Cardiopulmonary resuscitation outcome of out-of-hospital cardiac arrest in low-volume versus high-volume emergency departments: an observational study and propensity score matching analysis. Resuscitation 2011;82:32-9.

19. National Fire Agency. Statistical information [Internet]. Sejong: National Fire Agency [cited 2019 Nov 26]. Available from: https://www.nfa.go.kr/nfa/releaseinformation/statisticalinformation/main/.

20. Korean Statistical Information Service. Statistical database [Internet]. Daejeon: Statistics Korea [cited 2019 Nov 26]. Available from: http://kosis.kr/eng/statisticsList/statisticsListIndex. do?menuld=M_01_01\&twwcd = MT_ETITLECtparmTabld = M_01_01 .

21. Mochari-Greenberger $H$, Xian $Y$, Hellkamp AS, et al. Racial/ ethnic and sex differences in emergency medical services transport among hospitalized US stroke patients: analysis of the national get with the guidelines-stroke registry. J Am Heart Assoc 2015;4:e002099.

22. Sariyer G, Ataman MG, Akay S, Sofuoglu T, Sofuoglu Z. An 
analysis of Emergency Medical Services demand: time of day, day of the week, and location in the city. Turk J Emerg Med 2016;17:42-47.

23. Morrison $\sqcup$, Angelini MP, Vermeulen MJ, Schwartz B. Measuring the EMS patient access time interval and the impact of responding to high-rise buildings. Prehosp Emerg Care 2005; 9:14-8.

24. Ma J, Wang J, Zheng $W$, et al. Usage of ambulance transport and influencing factors in acute coronary syndrome: a cross- sectional study at a tertiary centre in China. BMJ Open 2017; 7:e015809.

25. Earnest A, Tan SB, Shahidah N, Ong ME. Geographical variation in ambulance calls is associated with socioeconomic status. Acad Emerg Med 2012;19:180-8.

26. Treno AJ, Gruenewald PJ, Lee JP, Remer LG. The Sacramento Neighborhood Alcohol Prevention Project: outcomes from a community prevention trial. J Stud Alcohol Drugs 2007;68: 197-207. 